\title{
PERSPECTIVAS DE LA FORMACIÓN PROFESIONAL EN COLOMBIA ENFOCADA EN EL SECTOR TURÍSTICO
}

\author{
PERSPECTIVES OF JOB TRAINING IN COLOMBIA \\ FOCUSED ON THE TOURISM SECTOR
}

Marysol Castillo-Palacio ${ }^{1 *}$, Alexander Zuñiga-Collazos ${ }^{2}$

\begin{tabular}{lr}
\hline${ }^{1}$ Docente tiempo completo. Universidad de Medellín, Colombia. & \\
& \\
\hline 2 Docente tiempo completo. Universidad de Medellín, Colombia. & Recibido: Enero 25 de 2015 \\
Aceptado: 30 de Julio de 2015 \\
& *Correspondencia del autor: E-mail: macastillo@udem.edu.co
\end{tabular}

\begin{abstract}
RESUMEN
Para los países latinoamericanos, el sector turismo representa una oportunidad de crecimiento, desarrollo económico y social. Sin embargo, en países como Colombia, la investigación y la formación de personal capacitado en este sector es aún deficiente (Castillo et al., 2011). Los objetivos del estudio son: identificar y analizar los programas académicos enfocados hacia el sector de turismo, ofertados en Colombia. La metodología utilizada para el estudio se centró en fuentes secundarias como el sistema general de información de la educación superior "SNIES" del Ministerio de Educación Nacional "MEN" de Colombia y las páginas web de las instituciones de educación superior colombianas que ofertan los programas en turismo. Finalmente, el estudio indica que los programas de pregrado en turismo son los de mayor oferta con respecto a los programas de posgrado en turismo.
\end{abstract}

Palabras Clave: turismo, formación profesional, educación superior, competitividad turística.

\begin{abstract}
Tourism sector represents for Latin American countries both a growth opportunity and an economic and social development. Nevertheless, scholarly research and skilled personnel training in this sector are still limited (Castillo et al., 2011). The aim of this study is to identify and analyze tourism academic programs which are offered in Colombia. The methodology focuses on secondary sources such as the higher education information system "SNIES", led by the Ministry of National Education "MEN" in Colombia, and the websites of the Colombian higher education institutions which offer these tourism programs. Finally, the results reveal that tourism are offered more in undergraduate programs than in postgraduate ones.
\end{abstract}

Keywords: tourism, job training, higher education, tourism competitiveness. 


\section{INTRODUCCIÓN}

Según la Organización Mundial del Turismo UNWOT (2012) el turismo es uno de los sectores económicos que mayor dinamismo ha tenido. Como lo reporta UNWTO (2013), las llegadas de turistas internacionales crecieron un 4,0\% en 2012 superando las 1.000 millones de llegadas de turistas a nivel mundial, por primera vez en la historia.

Según los resultados por regiones, Asia y el Pacifico registraron el más rápido crecimiento, con un incremento del $7 \%$ en las llegadas internacionales. África mostró un crecimiento de $6 \%$ o 3 millones más de turistas. Seguido por las Américas, la cual obtuvo un crecimiento del $5 \%$, equivalente a 7 millones de llegadas de turistas. Por otra parte, Europa registró un incremento del 3\%, o 18 millones más de llegadas. Contrario a estas regiones que han registrado un crecimiento en la llegada de turistas internacionales, Medio Oriente, aún sigue viéndose afectado por la situación política y económica de algunos de sus países, es así como registra un decrecimiento (-5\%).

En cuanto a la educación en el sector turismo, a nivel mundial, numerosos programas educativos son ofrecidos en esta gran industria. El número de puestos de trabajo en el turismo y la industria hotelera se estima entre los 120 millones a 231 millones en el mundo (Tribe 2002; Goeldner y Ritchie 2003; WTTC 2007; Fayos-Solá y Jafari 1996).

\section{El turismo en Colombia}

Según las proyecciones de población del Departamento Administrativo Nacional de Estadística de Colombia- DANE la población de Colombia para el 2012 es de 46.581 .823 habitantes, durante el mismo año recibió aproximadamente 2,175 millones visitantes extranjeros (UNWOT, 2013), lo que supone una intensidad turística (turistas extranjeros/habitantes) de 0,05 , valor que podría calificarse como muy bajo si se considera el extraordinario potencial que el turismo tiene en Colombia atendiendo a sus atractivos naturales y culturales. A su vez, en términos generales, la presión que el desarrollo turístico tiene sobre este país tampoco parece ser significativo, ya que la intensidad de la presión turística (turistas extranjeros/superficie en $\mathrm{Km}^{2}$ ) se sitúa en tan solo 1,90. Por otra parte, según el reporte del Foro Económico Mundial de 2013, el turismo genera en Colombia, directa e indirectamente, en torno al 5,1\% del Producto Interno Bruto (PIB) y $5,5 \%$ del empleo, porcentajes que también sugieren un mayor potencial de desarrollo turístico. Aunque se percibe un incremento con respecto a 2011 donde el turismo tuvo una participación de 4,3\% del PIB y $2,5 \%$ en cuanto al empleo.

El informe de Turismo de MinComercio (2013), indica que el número de llegadas de viajeros no residentes a diciembre de 2013 a Colombia (conformado por los extranjeros no residentes, pasajeros de cruceros internacionales y colombianos residentes en el exterior) es de 2,59 millones. Según el número de llegadas de viajeros no residentes en 2013, el país de procedencia corresponde principalmente y en su orden a Estados Unidos con 343.891 llegadas, Venezuela (239.284), Argentina (120.757), Ecuador (114.135), Perú (96.502) y España (89.535).

El reporte de la competitividad de los viajes y el turismo edición 2013 elaborado por el Foro Económico Mundial, revela que Colombia ocupa el puesto 84, de una lista de 140 países. Este análisis indica que Colombia no ha mejorado su nivel de competitividad turística relativa, ya que experimentó una pérdida de posiciones, ya que en 2011 ocupaba el puesto 77 entre 139 países, y en 2009 ocupaba el puesto 72 entre 133 países.

El potencial turístico de Colombia es evidente si se considera que la valoración del atractivo de sus recursos naturales y de su recursos culturales le permite ocupar los puestos 16 y 37 respectivamente, de un total de 140 países, dichos recursos pueden ser considerados atractivos turísticos, principalmente, para el turismo internacional (Castillo et al., 2011).

Colombia cuenta con extraordinarias ventajas comparativas vinculadas a sus recursos naturales y culturales (Zuñiga y Castillo, 2012a). Así mismo, los recursos humanos también contribuyen a la competitividad turística de Colombia al recibir valoraciones positivas con relación a la disponibilidad de personal cualificado y a los niveles de educación y formación, aunque según los últimos reportes de competitividad turística, Colombia ha perdido 8 posiciones de 2009 a 2013, en cuanto al pilar 11 relacionado con los recursos humanos.

Sin embargo, este aparente potencial de desarrollo turístico no está siendo adecuadamente aprovechado como consecuencia de un conjunto de circunstancias vinculadas a otras modalidades de ventajas compa- 
rativas y competitivas, como las condiciones de seguridad y sanitarias, las infraestructuras turísticas, el transporte, el marco regulador y la actividad empresarial. Este conjunto de factores también contribuyen a explicar en gran medida la escasa competitividad en precio de Colombia como destino turístico. No obstante, durante la pasada década, el turismo en Colombia y sus regiones ha experimentado un notable crecimiento (Zúñiga y Castillo, 2012b), y es evidente que el sector ha incrementado su aportación a la economía del país, en términos de generación de ingresos, creación de puestos de trabajo y reducción de la pobreza. $\mathrm{Y}$ en ese proceso de consolidación y crecimiento, es esencial la formulación de estrategias definidas para alcanzar un nivel de competitividad turística óptimo en cada departamento para promover un adecuado desarrollo de esta actividad en términos de competitividad y sostenibilidad, que permita maximizar los beneficios que ofrece el turismo para el bienestar de la población. El turismo es un sector estratégico para Colombia, siendo una fuente prioritaria de recuperación y desarrollo económico.

\section{La educación en turismo como un pilar fundamen- tal de la competitividad turística de un país}

El sector turístico es un contribuyente importante a las economías de muchos países. Los países necesitan una fuerza laboral educada, capacitada y comprometida, para que este sector tenga éxito (Koyuncu et al., 2008). El nivel educativo enfocado en turismo, es un factor preponderante, y puede tener un efecto directo sobre la competitividad turística de los países, este es uno de los indicadores utilizados por el Foro Económico Mundial para medir la competitividad turística de un país (WEF, 2013). En la literatura sobre turismo, el concepto de competitividad turística ha evolucionado a lo largo del tiempo. Así por ejemplo, D'Hartserre (2000) definió la competitividad turística como la capacidad de un destino para mantener y/o mejorar su posición en el mercado a través del tiempo. Por su parte, Hassan (2000) vinculó la competitividad de un destino con su capacidad para crear e integrar productos de mayor valor añadido que sustenten sus recursos, mientras mantiene la posición en el mercado con relación a sus competidores. La competitividad es un concepto relativo y multidimensional (Porter, 1990).

Existe la necesidad de tener personal altamente capacitado con altos estándares de calidad, los diseños de programas tanto de pregrado como de postgrado deben responder a diferentes tipos de necesidades del sector, de la comunidad académica y debe contar con el apoyo del estado (Ring et al., 2009). De igual manera, Wu et al. (2014) sugieren que la cantidad de programas en turismo es importante, pero en algunos países es necesario mejorar la calidad de dichos programas. Además, los programas académicos, en especial los programas de postgrado, deben tener un componente altamente investigativo, que permita identificar posibles soluciones a las problemáticas del sector. El estudio realizado por Severt et al. (2009), presenta un ranking de los 100 mejores programas en turismo en el mundo, y describe como uno de los indicadores clave de reconocimiento, el grado de investigación, publicación, divulgación e impacto en el sector de cada programa. De acuerdo a Ayoun et al. (2010), la formación del talento humano en turismo debe darse en un ambiente cada vez más globalizado, las redes de investigación deben internacionalizarse compartiendo experiencias y desarrollando nuevo conocimiento, además, la productividad de la investigación es un factor muy importante, para el desarrollo del sector en los países.

Lee y Law (2011) estudiaron como la productividad de la investigación presenta relación directa con la oferta de programas de doctorado, y con dos factores especialmente identificados: el tamaño de las facultades de turismo, y el nivel de autonomía que tengan las instituciones y sus profesores para que puedan desarrollar su actividad investigativa. Sobre la calidad y procesos de enseñanza en los programas de pregrado de turismo Kim y Davies (2014) sugieren que una enseñanza efectiva en los cursos de turismo de pregrado requieren de una mayor colaboración entre los procesos de enseñanza y aprendizaje para maximizar la alta calidad en el aprendizaje de los estudiantes. Lo cual incluye, objetivos de aprendizaje, aprendizaje autodirigido, una guía clara sobre cómo desarrollar una actividad, y retroalimentación sobre los procesos de aprendizaje.

Algunos estudios demuestran, que en algunos países, como los pertenecientes al continente asiático, están desarrollando mayores programas a nivel pregrado y postgrado en el sector turístico y en la gestión del turismo, lo cual podría permitir incrementar, aún más, su competitividad y crecimiento turístico. Kim et al. (2008), estudiaron los principales factores de motivación de los estudiantes coreanos, para realizar este tipo de estudios, e identificaron que la auto-realiza- 
ción, oportunidad de empleo y la experiencia en el extranjero, fueron los principales motivadores para los estudiantes de pregrado, mientras que los estudiantes de postgrado eligieron la auto-realización y logro educativo, como los dos motivadores más importantes.

De igual manera, la identificación y desarrollo de valores o principios, las proyecciones de futuro de los programas, que realmente interesen y desarrollen las instituciones y los estudiantes, son de vital importancia para el óptimo desarrollo de los programas de turismo (Padurean y Maggi, 2011). Wu et al. (2014) realizaron una investigación sobre los perfiles de los programas de pregrado de educación en turismo en términos de las afiliaciones disciplinarias, las especializaciones de los programas y el contexto académico de los miembros del profesorado que enseñan turismo, hallaron entonces que las principales afiliaciones disciplinarias fueron gestión, geografía y geología, sociología y humanidades, y economía. Las principales especializaciones del programa fueron gestión del turismo, gestión de la hospitalidad y la planeación del turismo. Se observa que los programas de pregrado en turismo en China están enfocados a la planeación, la cual es requerida por el rápido crecimiento del turismo en este país. Horng y Lee (2005) recomiendan que la industria, el gobierno y la academia en Taiwan, y en los países en general, deberían trabajar juntos para el desarrollo y las decisiones sobre las reformas futuras a la educación en turismo y en hospitalidad.

\section{El capital humano y la competitividad}

Gibbons y Johnston (1974), plantean que la educación universitaria es de gran importancia para los directores, debido a que permite desarrollar mejores habilidades para la solución de los problemas. De igual manera, Gu et al. (2007), sustentan que una fuerza laboral bien preparada requiere incluir la gestión profesional y liderazgo, y es allí donde radica la importancia de la participación de la educación superior.

Cañibano-Sanchez (2005), sugiere que los gobiernos nacionales, deben centrarse en dotar a sus economías de mayores niveles de capital humano, el cual estará inmerso en un sector productivo, aportando productividad y competitividad al mismo.

Específicamente en los programas de educación, en este caso los enfocados en turismo, tienen elementos importantes, entre los que se encuentra el diseño del plan de estudios, el cual requiere una sociedad turística, no solo para los negocios, sino para los grupos de interés. Debe existir, entonces un equilibrio entre la práctica y la técnica (Tribe, 2001). Los beneficiados de un plan de estudios sobre turismo bien diseñado son los empleadores y turistas, así como los graduados de estos programas (Gu et al., 2007).

\section{Desarrollo de la educación en turismo}

La educación en turismo inició como cursos de entrenamiento en sub-sectores específicos. Posteriormente, estos cursos dieron paso a las escuelas técnicas y vocacionales, las cuales han estructurado programas de pregrado y postgrado. Debido al continuo crecimiento del sector turismo a nivel mundial, se ha generado la necesidad de profesionales educados (Busby y Fiedel, 2001; Ernatawi, 2003). En la actual era de la información, la cual ha dado paso a la globalización y a un entorno cambiante, donde se requiere una rápida y adecuada toma de decisiones, pensamiento crítico y analítico, creatividad, innovación, solución de problemas y adaptación a los cambios, genera la necesidad de graduados bien preparados. Esto incluye una concentración sobre habilidades genéricas, las cuales son descritas como "aquellas habilidades transferibles que son esenciales para la empleabilidad en algunos niveles" (Raybould y Wilkins 2005, p. 204).

\section{Metodología}

Las Instituciones de Educación Superior (IES) son las entidades, que de acuerdo a las normas legales colombianas, son reconocidas oficialmente como prestadoras del servicio público y privado de la educación superior en el territorio de este país. Se clasifican según su carácter académico (clasificación A) y según su naturaleza jurídica (clasificación B). Según su carácter académico, las Instituciones de Educación Superior (IES) se clasifican en: Instituciones Técnicas Profesionales, Instituciones Tecnológicas, Instituciones Universitarias o Escuelas Tecnológicas, Universidades. El carácter académico de universidad lo pueden alcanzar por mandato legal (Art. 20 Ley 30 de la Constitución Colombiana) las instituciones que, teniendo el carácter académico de instituciones universitarias o escuelas tecnológicas, cumplan los requisitos indicados en el artículo 20 de la Ley 30 de 1992, los cuales están desarrollados en el Decreto 1212 de $1993^{1}$.

Para determinar la oferta de los programas que tienen

${ }^{1}$ Ministerio de Educación Nacional, República de Colombia. Instituciones de Educación Superior. www.mineducacion.gov.co, último acceso: marzo de 2014. 
como eje central el sector del turismo en Colombia, se realizó una búsqueda en fuentes secundarias, utilizando como principal fuente de información todos los programas de niveles académicos: 1. Posgrados, los cuales se dividen en: Especializaciones, Maestrías y Doctorados, y 2. Profesional Universitario, que se encuentran registrados en el sistema general de información de la educación superior "SNIES" del Ministerio de Educación Nacional "MEN" de Colombia, la consulta fue realizada en el mes de Marzo de 2014; las palabras clave de búsqueda fueron: "turismo", "turística", "turísticos" y "turísticas" en el icono "nombre del programa". El resultado obtenido fue de 75 programas, de los cuales 14 (18.67\%) se encontraron inactivos. Por lo tanto, en el momento de la búsqueda existen registrados $61(81.33 \%$ ) programas activos de formación en turismo a estos niveles de educación superior en Colombia.

El análisis de resultados se realizó con los 61 programas activos en turismo ofertados en Colombia, se desarrolló utilizando las siguientes variables: 1. El tipo de instituciones de educación superior donde se imparten los programas, las cuales, según su carácter académico, se clasifican en: Instituciones Técnicas Profesionales, Instituciones Tecnológicas, Instituciones Universitarias o Escuelas Tecnológicas y Universidades. 2. Se identificó si la institución era privada u oficial, 3. Se analizaron los niveles académicos de los programas activos ofertados: Pregrado: Profesional Universitario y Posgrado: Especialización, Maestría y Doctorado. 4. Se identificó la metodología en la que ofertan los programas: Presencial, Distancia (Tradicional), Distancia (Virtual). 5. Se realizó un análisis comparativo de la ubicación geográfica por departamento donde se ofertan los programas y sus niveles académicos. Y finalmente, 6 . Se consultó la información reportada de cada programa en las páginas web de las instituciones de educación superior colombianas, que aparecían como responsables de la oferta de los programas en turismo analizados, para determinar cuáles eran los perfiles de los programas ofertados, las líneas de énfasis o electivas ofrecidas por estos programas y si estos incluyen en sus planes de estudio la formación en idiomas extranjeros.

\section{Análisis de resultados \\ Programas en turismo ofertados en Colombia}

La Tabla 1 muestra la participación de las diferentes Instituciones de Educación Superior, que ofertan los programas de turismo a nivel de profesional universitario y posgrado en Colombia, donde se muestra que las universidades tienen una mayor oferta de este tipo de programas, ya que representan el $65.57 \%$ con 40 programas académicos ofertados, seguido por las instituciones universitarias o escuelas tecnológicas con $27.87 \%$ (17 programas ofertados), continúan las instituciones técnicas profesionales con 4.92\% (3 programas ofertados) y finalmente, las instituciones tecnológicas tiene una participación de $1.64 \%$ (1 programa). De los 61 programas analizados. La oferta es dominada principalmente por instituciones de carácter privado equivalente al $70.49 \%$ (43 instituciones de educación superior) y el 29.51\% (18 Instituciones) son de carácter oficial.

Tabla 1. Tipo de Institución de Educación Superior que oferta programas de turismo a nivel de profesional universitario y posgrado en Colombia.

\begin{tabular}{ccc}
\hline Tipo de Institución & Número de Programas & Frecuencia Porcentaje (\%) \\
\hline Institución Técnica Profesional & 3 & 4.92 \\
Institución Tecnológica & 1 & 1.64 \\
Institución Universitaria o Escuela Tecnológica & 17 & 27.87 \\
Universidad & 40 & 65.57 \\
Programas ofertados en Institución Privada & 43 & 70.49 \\
Programas de Institución Pública & 18 & 29.51 \\
Total de programas de Turismo en Colombia & $\mathbf{6 1}$ & $\mathbf{1 0 0 \%}$ \\
\hline
\end{tabular}

Fuente: Elaboración propia a partir de los resultados de la búsqueda en SNIES del MEN de Colombia, marzo de 2014.

En la Tabla 2 se observa, que de acuerdo a los niveles de formación analizados, el mayor número de programas relacionados con el sector turismo, ofertados en Colombia, son de nivel profesional universitario con un $75.41 \%$ (46 programas), seguido por los programas de posgrado a nivel de especialización con un
$21.31 \%$ (13 programas), a continuación se ubican los programas de posgrado a nivel de maestría con 3.28\% equivalente a 2 programas. Finalmente en este estudio no se pudo determinar la oferta de ningún programa de posgrado a nivel de doctorado que tuviese relación con el sector turístico, y que estuviese ofertado y acti- 
Perspectivas de la formación profesional en Colombia en el sector turístico. Castillo y Zuñiga.

vo en el sistema de educación superior en Colombia. Además, en estos resultados, se observa, que en general continúa siendo la modalidad presencial la de mayor acogida, equivalente a un $85.25 \%$ (52 programas). La metodología de distancia tradicional presenta una participación del $8.2 \%$ (5 programas), mientras que la metodología de distancia virtual tiene una participación equivalente al $6.56 \%$ (4 programas), lo que demuestra que la modalidad virtual, tiene una tendencia de crecimiento, ya que la modalidad de distan- cia virtual es relativamente nueva comparada con la tradicional, y ésta podría ser parte de una estrategia para ampliar la cobertura, siempre y cuando el diseño de programas con este tipo de modalidad satisfaga las necesidades no solo del sector si no de los estudiantes virtuales, de acuerdo a Lu y Chen (2011), uno de los factores clave, es el buen manejo de los momentos sincrónicos y asincrónicos virtuales, que esta modalidad permita desarrollar.

Tabla 2. Nivel académico y metodología en que se ofertan los programas de turismo en Colombia.

\begin{tabular}{|c|c|c|c|c|c|}
\hline $\begin{array}{l}\text { Nivel Académico/ } \\
\text { Metodología }\end{array}$ & Número de Programas (\#) & $\begin{array}{c}\text { Porcentaje } \\
(\%)\end{array}$ & Metodología & $\begin{array}{c}\text { Número } \\
\text { (\#) }\end{array}$ & $\begin{array}{c}\text { Porcentaje } \\
(\%)\end{array}$ \\
\hline \multirow{3}{*}{$\begin{array}{l}\text { Nivel Profesional } \\
\text { Universitario }\end{array}$} & 46 & 75.41 & Presencial & 40 & 65.57 \\
\hline & & & Distancia Tradicional & 4 & 6.56 \\
\hline & & & Distancia Virtual & 2 & 3.28 \\
\hline \multirow[t]{3}{*}{ Especialización } & 13 & 21.31 & Presencial & 10 & 16.39 \\
\hline & & & Distancia Tradicional & 1 & 1.64 \\
\hline & & & Distancia Virtual & 2 & 3.28 \\
\hline \multirow[t]{3}{*}{ Maestría } & 2 & 3.28 & Presencial & 2 & 3.28 \\
\hline & & & Distancia Tradicional & - & - \\
\hline & & & Distancia Virtual & - & - \\
\hline \multirow[t]{3}{*}{ Doctorado } & 0 & - & Presencial & - & - \\
\hline & & & Distancia Tradicional & - & - \\
\hline & & & Distancia Virtual & - & - \\
\hline \multirow[t]{4}{*}{ Total Programas } & 61 & $100 \%$ & Total Programas & 61 & $100 \%$ \\
\hline & \multicolumn{2}{|c|}{ Total Presencial } & & 52 & 85.25 \\
\hline & \multicolumn{2}{|c|}{ Total Distacia Tradicional } & & 5 & 8.20 \\
\hline & \multicolumn{2}{|c|}{ Total Distacia Virtual } & & 4 & 6.56 \\
\hline
\end{tabular}

Fuente: Elaboración propia a partir de los resultados de la búsqueda en SNIES del MEN de Colombia, 2014.

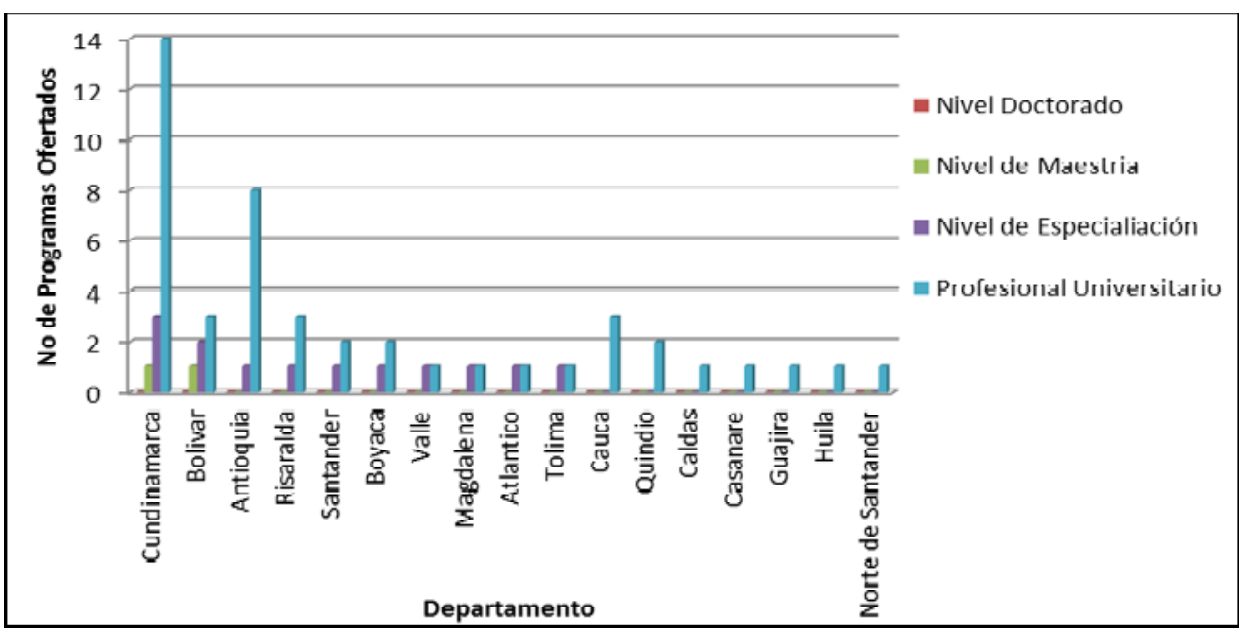

Figura 1. Ubicación geográfica y nivel académico de los programas en turismo ofertados en Colombia

Fuente: Elaboración propia a partir de los resultados de la búsqueda en SNIES del MEN de Colombia 2014. 
La figura 1, muestra la ubicación geográfica de los programas analizados, activos y ofertados en Colombia, donde se puede observar que el mayor número de programas ofertados tanto de postgrado como de pregrado se encuentra ofertado en el departamento de Cundinamarca, además los departamentos que siguen teniendo en cuenta el nivel de formación son Bolívar, Antioquia, Risaralda, Santander, Boyacá, Valle, Magdalena, Atlántico, Tolima, Cauca, Quindío, Caldas, Casanare, Guajira, Huila y Norte de Santander.

El departamento que presenta mayor participación en la oferta de programas profesionales universitarios y de posgrado es Cundinamarca con un (1) programa de posgrado a nivel de maestría, 3 posgrados a nivel de especialización, además se ofertan 14 programas a nivel de profesional universitario. Por otro lado, y de acuerdo al nivel de formación, el departamento de
Bolívar es el que sigue en la lista, al ofertar un (1) programa de posgrado a nivel de maestría, 2 a nivel de especialización y 3 programas a nivel de profesional universitario, y el tercer lugar por nivel de formación lo ocupa el departamento de Antioquia ofertando un (1) programa a nivel de especialización y 8 programas a nivel de profesional universitario.

Tipos de electivas o líneas de énfasis en los programas de pregrado en turismo ofertados en Colombia

Como se observa en la figura 2, la mayor parte de las electivas ofertadas en los programas académicos de pregrado enfocados en turismo, son las que las instituciones denominan electivas profesionales, seguido por la electiva ecoturismo o turismo de naturaleza con $7.78 \%$ ( 7 electivas), luego continua administración en empresas turísticas con $6.67 \%$.

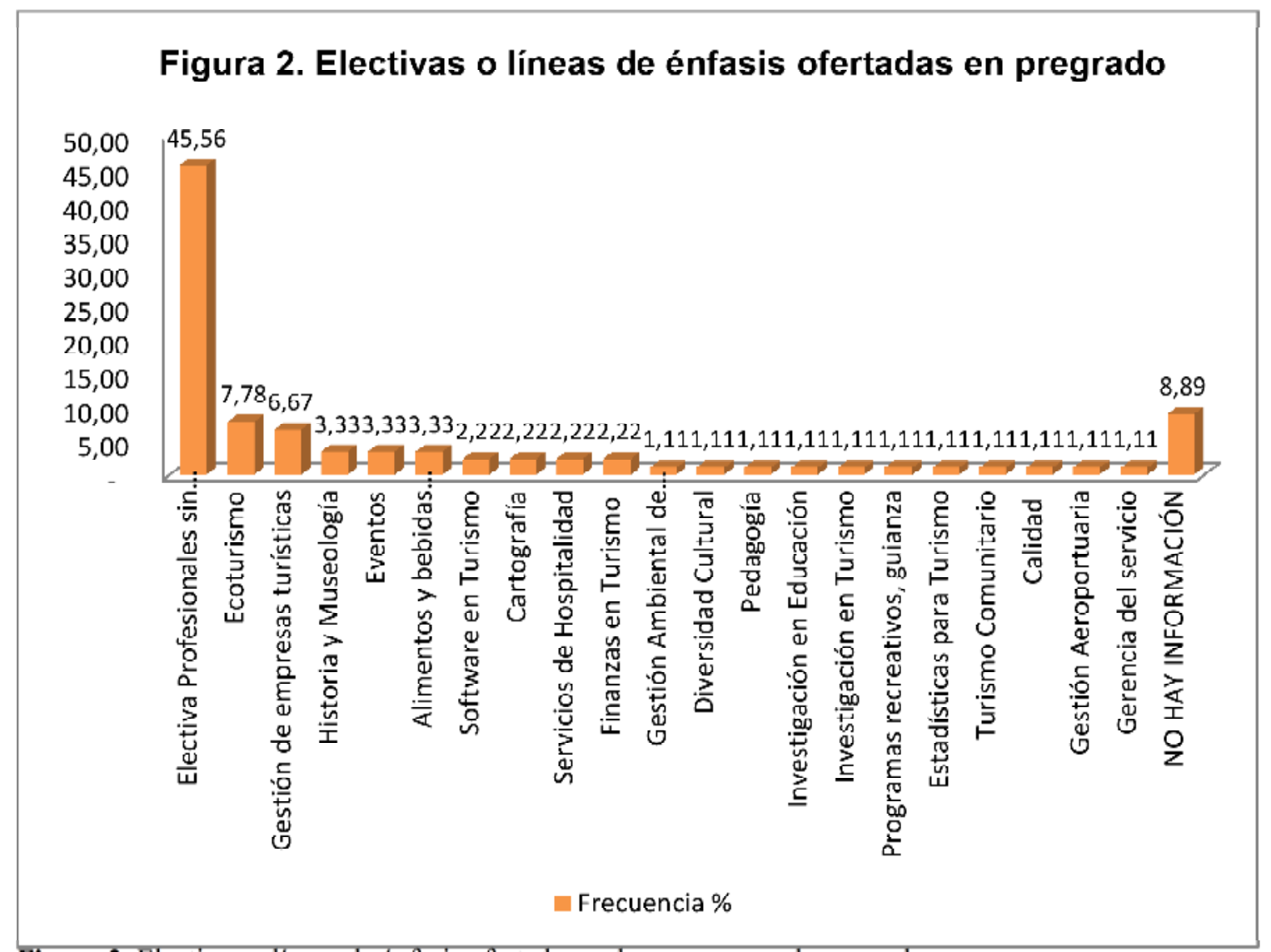

Figura 2. Electivas o líneas de énfasis ofertadas en los programas de pregrado

Fuente: Elaboración propia a partir de los resultados de la búsqueda en las páginas Web de cada institución de educación superior, Marzo de 2014.

Perfiles de los programas de pregrado sobre turismo ofertados en Colombia

La tabla 3 muestra que en general, los perfiles de los programas a nivel profesional universitario ofertados en Colombia se concentran en el área de las ciencias administrativas, con una participación del 77.05\% (47 programas de los 61 ofertados), seguido por investigación y consultoría con $8.2 \%$ (5 programas de 61$)$, y 
finalmente se encuentra educación con 1.64\% (1 programa de 61). Por otro lado, el $13.11 \%$ equivalente a 8 programas de 61 , no presentaban información de ningún tipo en sus páginas web.

Tabla 3. Perfiles de los programas enfocados en turismo en Colombia.

\begin{tabular}{|c|c|c|c|c|}
\hline Área del programa & Frecuencia \% & Perfil del programa & Número de programas & Frecuencia \% \\
\hline \multirow{4}{*}{ Ciencias de la administración } & \multirow{4}{*}{77.05} & Gestión & 31 & 50.82 \\
\hline & & Planeación & 8 & 13.11 \\
\hline & & Dirección & 5 & 8.20 \\
\hline & & Emprendimiento & 3 & 4.92 \\
\hline Investigación y consultoría & 8.20 & Investigacióny consultoría & 5 & 8.20 \\
\hline Educación & 1.64 & Educación & 1 & 1.64 \\
\hline No hay información disponible & 13.11 & $\begin{array}{l}\text { No hay información dispo- } \\
\text { nible }\end{array}$ & 8 & 13.11 \\
\hline Total & 100.00 & Total & 61 & 100.00 \\
\hline
\end{tabular}

Fuente: Elaboración propia a partir de los resultados de la búsqueda en las páginas Web de cada institución de educación superior, Marzo de 2014.

Áreas de formación de los programas de turismo ofertados a nivel de postgrado

Cada programa de postgrado agrupa diversas áreas de formación, y el número de programas que tienen información disponible en las páginas web de cada entidad educativa son solo 6 de los 15 registrados en SNIES. Como lo indica la tabla 4, la mayoría de los programas de postgrado están orientados a la administración (5 programas), seguido por la planificación turística, el mercadeo turístico, el desarrollo sostenible y la investigación, cada una de estas áreas, presentes en 3 programas de los 6 programas consultados. Se hallaron dos programas de especialización que incluyen dentro de sus áreas de formación el turismo especializado, específicamente orientado a turismo de salud, turismo rural y turismo de aventura. Finalmente, solo un programa de postgrado, en este caso una maestría, incluye la legislación dentro de sus áreas de formación.

Tabla 4. Áreas de Formación de los programas de postgrado en turismo*

\begin{tabular}{lc}
\hline \multicolumn{1}{c}{ Áreas de formación } & Número de programas \\
\hline Planificación Turística & 3 \\
Administración/Gerencia/ & 5 \\
Dirección & \\
Mercadeo Turístico & 3 \\
Investigación & 3 \\
Desarrollo sostenible & 3 \\
Economía del turismo & 2 \\
Turismo especializado & 2 \\
Legislación & 1 \\
Total programas de post- & $\mathbf{6}$ \\
grado consultados & \\
\hline
\end{tabular}

*El número de programas consultados según la disponibilidad de la información en las páginas web oficiales de cada institución educativa es 6 , pero cada programa incluye diversas áreas de formación en el plan de formación.

Fuente: Elaboración propia a partir de los resultados de la búsqueda en las páginas web de cada institución de educación superior, Marzo de 2014.

\section{Una segunda lengua extranjera en los planes de} formación de pregrado en turismo

Ring et al. (2009) realizaron un análisis a 64 programas de turismo de pregrado en inglés, en los Estados Unidos, Canadá, Reino Unido, España y otros países europeos y del resto del mundo, concluyeron que dentro del contenido de los planes de estudio lo más importante son las pasantías, la gestión y las lenguas extranjeras.

Existen 33 programas de pregrado en turismo que se promocionan a través de las páginas web oficiales de las instituciones de educación superior, de los cuales 17 programas incluyen asignaturas de formación en una segunda lengua extranjera en sus planes de formación, lo que equivale al $52 \%$ del total de los programas consultados; 8 programas no incluyen niveles de formación en un segundo idioma extranjero y 8 programas no presentan información sobre sus planes de formación, por lo cual no se puede identificar si incluyen o no formación en un segundo idioma. De los 17 programas que incluyen asignaturas de formación en una segunda lengua extranjera, 16 definen claramente que la segunda lengua extranjera es inglés, donde uno de estos programas incluye, además formación en francés. Por otra parte, hay un (1) programa que indica niveles de formación en idioma extranjero pero no indica cual es el idioma en el que 
se hace énfasis. La mayoría de estos programas desarrolla la formación en segunda lengua extranjera en dos niveles. Por otra parte, en cuanto al número de créditos asignados a cada nivel de formación en una segunda lengua extranjera, en la mayoría de los casos, se asignan 2 créditos (en 6 programas).

\section{Conclusiones}

Según lo analizado, se concluye que de acuerdo a los niveles de formación analizados, el mayor número de programas relacionados con el sector turístico en Colombia, los ofertan las universidades teniendo una participación de $65.57 \%$ (40 programas académicos). De los 61 programas analizados, las instituciones de carácter privado ofertan la mayoría de los programas con un $70.49 \%$ frente al $29.51 \%$ que corresponde a las instituciones de carácter oficial.

En cuanto a la ubicación geográfica el mayor de número de programas sobre turismo a nivel de pregrado (profesional universitario) y postgrado (especialización y maestría) se ofertan en el departamento de Cundinamarca. Respecto a las líneas de énfasis, se logró determinar que la mayor parte de las electivas son las que las instituciones denominan electivas profesionales con un $44.56 \%$ (41 electivas), seguido por la electiva ecoturismo o turismo de naturaleza con $7.78 \%$ ( 7 electivas), luego continua administración en empresas turísticas con $6.67 \%$ (6 electivas).

Además, los resultados de este estudio demostraron que en cuanto a los perfiles de los programas a nivel profesional universitario, se concentran en el área de las ciencias administrativas $(77.05 \%)$, seguido por investigación y consultoría con $8.2 \%$, y finalmente se encuentra educación con $1.64 \%$ (1 programa de 61 ). Sobre la formación en una segunda lengua extranjera, el $52 \%$ de los programas de pregrado en turismo consultados incluyen este tipo de formación en sus planes de formación, donde el idioma inglés es predominante.

Por otro lado, el $13.11 \%$ equivalente a 8 programas de 61 tipos de perfiles analizados, pertenecientes a 46 programas a nivel profesional universitario, no presentaban información de ningún tipo en sus páginas web, esto puede ser debido a que aunque las instituciones tenían registro calificado activo del programa ante la base de datos SNIES de MEN, estos programas podrían no estar ofertándose al mercado, reduciendo así el número de programas a nivel profesional universitario realmente ofertados en este país a 38 programas. En cuanto al nivel de postgrado la principal área de formación presente en el $83 \%$ de los programas consultados, es la administración/gerencia/dirección, seguido por la planeación turística, el mercadeo turístico, el desarrollo sostenible y la investigación.

Los programas universitarios de pregrado y posgrado relacionados con la formación en turismo van en aumento. Por otra parte, el gobierno nacional y los gobiernos locales han incentivado el desarrollo del turismo y la formación en algunas regiones del país. Sin embargo, se observa que a nivel de posgrado el número de programas ofertados es bajo, en el caso específico de los programas de doctorado en turismo, actualmente no se oferta ninguno en Colombia, y es requerido fortalecer el número de programas y su calidad de acuerdo al crecimiento constante del sector en el ámbito nacional.

Dentro de las líneas futuras de investigación, se propone realizar un análisis más profundo acerca de los contenidos de los programas de turismo. Además, también se pueden desarrollar estudios sobre la percepción de los estudiantes respecto a la relación entre la enseñanza y la investigación, así como estudiar el contexto académico del grupo de profesores de los programas de pregrado y posgrado en turismo.

Una limitación del estudio, se relaciona con que pueden existir algunos programas que no fueron tenidos en cuenta en el desarrollo del estudio, debido a que no se encontraron registrados en el SNIES, en el momento de la consulta o simplemente en su denominación las palabras clave de búsqueda no están presentes. 


\section{BIBLIOGRAFÍA}

\section{- Articulos}

Ayoun, B., Johnson, M. K., Vanhyfte, M. y O'Neill, M (2010). A Comparison Study of U.S. and Non-U.S. Education Internationalization Practices of Hospitality and Tourism Programs. Journal of Teaching in Travel \& Tourism, 10(1), 335-361.

- Busby, G. y Fiedel, D. (2001). A Contemporary Review of Tourism Degrees in the United Kingdom. Journal of Vocational Education and Training, 53(4), 501-522.

- D'Hauteserre, A.M. (2000). Lessons in managed destination competitiveness: The case of Foxwoods Casino Resort. Tourism Management, 21(1), 23-32.

- Ernatawi, D.B. (2003). Stakeholder's View on Higher Tourism Education. Annals of Tourism Research, 30(1), 255-58.

- Gibbons, M. y Johnston, R. (1974). The roles of science in technological innovation. Research Policy, 3(3), 220-242.

- Gu, H., Kavanaugh R.R. y Cong, Y. (2007). Empirical studies of tourism education in China. Journal of Teaching in Travel \& Tourism, 7(1), 3-24.

- Hassan, S.S. (2000). Determinants of Market Competitiveness in an Environmentally Sustainable Tourism Industry. Journal of Travel Research, 38(3), 239-245.

- Horng, J.S., Lee. M.H. (2005). Tourism and Hospitality Higher Education in Taiwan: Past, Present and Future. Journal of Teaching in Travel \& Tourism, 5(1), 167-196.

- Kim, A.K. y Davies, J. (2014). A Teacher's Perspective on Student Centred Learning: Towards the Development of Best Practice in an Pregraduate Tourism Course. Journal of Hospitality, Leisure, Sport \& Tourism Education, 14(1), 6-14.

- $\quad$ Kim, S.S., Lee, M.J. y Chon, K. (2008). Study Motivations and Study Preferences in the Korean Hospitality and Tourism Field. Journal of Marketing for Higher Education, 18(2), 216-239.

- Koyuncu, M., Burke, R.J., Fiksenbaum, L. y Demirer, J. (2008). Predictors of Commitment to Careers in the Tourism Industry. Anatolia: An International Journal of Tourism and Hospitality Research, 19(2), 225-236.

- Lee, H. y Law, R. (2011). Research productivity and institutional characteristics of hospitality and tourism programs. Journal of Travel \& Tourism Marketing, 28(1), 432-450.

- $\quad$ Lu, C.Y. y Chen, B.T. (2011). The Potential for Active Online Learning in Taiwanese Tourism Degree Programs Based on Online Educational Experiences of Graduate Students. Journal of Teaching in Travel \& Tourism, 11(3), 271-288.

- $\quad$ Padurean, L. y Maggi, R. (2011). TEFI Values in Tourism Education: A Comparative Analysis. Journal of Teaching in Travel \& Tourism, 11(1), 24-37.

- $\quad$ Raybould, M. y Hugh W. (2005). Over Qualified and Under Experienced: Turning Graduates into Hospitality Managers. International Journal of Contemporary Hospitality Management, 17(3), 203-16.

- $\quad$ Ring, A., Dickinger, A. y Wöber, K. (2009). Designing the Ideal Pregraduate Program in Tourism. Expectations from Industry and Educators. Journal of Travel Research, 48 (1), 106-121.

- $\quad$ Severt, D.E., Tesone, D.V., Bottorff, T.J. y Carpenter, M.L. (2009). A world ranking of the top 100 hospitality and tourism programs. Journal of Hospitality \& Tourism Research, 33(4), 451-470.

- Tribe, J. (2001). Research paradigms and the tourism curriculum, Journal of Travel Research, 39(4), $442-448$.

- Tribe, J. (2002). The Philosophic Practitioner. Annals of Tourism Research, 29(2), 338-357.

- Wu, B., Morrison, A.M., Yang, J.K., Zhou, J.L. y Cong, L. (2014). Cracks in the ivory tower? A surveybased analysis of pregraduate tourism education and educators in China. Journal of Hospitality, Leisure, Sport \& Tourism Education, 14(1) 26-38.

- Zuñiga-Collazos, A. y Castillo-Palacio, M. (2012a). Análisis Académico y Profesional del Desarrollo Turístico en Colombia. (2009-2010). Magazín Empresarial, 16(1), Editorial Universidad Santiago de Cali, Colombia.

- Zuñiga-Collazos, A. y Castillo-Palacio, M. (2012b). Caracterización de la Formación en Turismo, como uno de los Pilares de la Competitividad Turística en Colombia. Anuario Turismo y Sociedad, 13(1), 227-249. 


\section{Libros:}

- Fayos-Solá, E. (1997). An Introduction to TedQual: A Methodology for Quality in Tourism Education and Training. Madrid, España: World Tourism Organization.

- Goeldner, C.R. y Brent J.R. (2003). Tourism: Principles, Practices, Philosophies. New York, USA: John Wiley \& Sons.

- Porter, M.E. (1990). The competitive advantage of nations. New York, USA: Ed. Free Press.

- Ritchie, J.R.B. y Crouch, G.I. (2003). The competitive destination: A sustainable tourism perspective. Oxon: CABI Publishing.

\section{Informes Técnicos:}

- Cañibano-Sanchez, C. (2005). Capital humano: factor de innovación, competitividad y crecimiento. Sexto Congreso de Economía de Navarra: Competitividad y Crecimiento Económico. Pamplona, España.

- Castillo-Palacio, M., Zúñiga-Collazos, A. y Chim-Miki, A.F. (2011). Análisis de la Investigación Científica Internacional Sobre Turismo y la Competitividad del Sector Turístico en Argentina, Brasil, Chile y Colombia. Encuentro Internacional de Investigadores en Administración. Universidad Externado de Colombia, Bogotá, Colombia.

- Departamento Administrativo Nacional de Estadística (DANE), Colombia. Población estimada desde 1985 a 2020. Recuperado: https://www.dane.gov.co (Ultimo acceso: 07/Marzo/ 2014).

- United Nations Word Organization Tourism UNWOT (2013), Tourism Highlights 2013.

- United Nations Word Organization Tourism UNWOT (2012), World Tourism Barometer, 2011 International Tourism Results and Prospects for 2012.

- Ministerio de Comercio, Industria y Turismo de Colombia (2013), Informe del Turismo en Colombia a Diciembre/2013.

- World Economic Forum WEF (2013). The Travel \& Tourism Competitiveness Report: Reducing Barriers to Economic Growth and Job Creation 2013.

- World Economic Forum WEF (2011). The Travel \& Tourism Competitiveness Report 2011.

- World Economic Forum WEF (2009). The Travel \& Tourism Competitiveness Report: Reducing Barriers to Economic Growth and Job Creation 2009.

- World Travel and Tourism Council WTTC (2007). Tourism satellite accounting. Recovered:http:// www.wttc.org/eng/Research/Tourism_Satellite_Accounting/index.php 